\title{
Hierarchical Absorbing Interface Conditions for Wave Propagation on Non-Uniform Meshes
}

\author{
Shuyang Dai ${ }^{1,2}$, Zhiyuan Sun ${ }^{2}$, Fengru Wang ${ }^{1}$, \\ Jerry Zhijian Yang ${ }^{1,2, *}$ and Cheng Yuan ${ }^{3}$ \\ ${ }^{1}$ School of Mathematics and Statistics, Wuhan University, Wuhan 430072, \\ P.R. China \\ ${ }^{2}$ Computational Science Hubei Key Laboratory, Wuhan University, Wuhan \\ 430072, P.R.China \\ ${ }^{3}$ Three Gorges Mathematical Research Center, China Three Gorges University, \\ Yichang 443002, Hubei, China
}

Received 2 August 2021; Accepted (in revised version) 8 December 2021

\begin{abstract}
In this paper, we propose hierarchical absorbing interface conditions to solve the problem of wave propagation in domains with a non-uniform space discretization or grid size inhomogeneity using Padé Via Lanczos (PVL) method. The proposed interface conditions add an auxiliary variable in the wave system to eliminate the spurious reflection at the interface between regions with different mesh sizes. The auxiliary variable with proper boundary condition can suppress the spurious reflection by cancelling the boundary source term produced by the space inhomogeneity in variational perspective. The new hierarchical interface conditions with the help of PVL implementation can effectively reduce the degree of freedom in solving the wave propagation problem.
\end{abstract}

AMS subject classifications: 65K10, 65N22, 35L05

Key words: Wave equation, absorbing interface condition, spurious reflection, Padé via Lanczos.

\section{Introduction}

Numerical methods such as finite element method (FEM) and finite difference method (FDM) are widely used for simulating various physical phenomenon such as structural response of materials, evolution of phase transformation and wave propagation in medium. Many scientific discoveries and industry advances rely on the development of modern numerical techniques. In most numerical methods, there is an im-

${ }^{*}$ Corresponding author. Email addresses: shuyang_dai@whu.edu.cn (S. Dai), sun_zhiyuan@iapcm.ac.cn (Z. Sun), wangfr@whu.edu.cn (F. Wang), zjyang.math@whu.edu.cn (J.Z. Yang), yuancheng@whu.edu.cn (C. Yuan) 
portant question on how to determine the numerical spatial resolution or discretization mesh size in the actual implementation of simulations. Although if increasing the numerical spatial resolution or equivalently decreasing the discretization mesh size could provide more information and solve the problems with a higher precision, however, the computational cost due to the unified high spatial resolution in the whole domain of calculation is consuming. Since in most realistic simulations, the information we are interested in is only bounded in a small region, and the whole computational domain is relatively large, it is important to develop an unified numerical framework that could couple different models with various spatial resolution or discretization mesh size $[8,9,18,19]$. This numerical framework could increase the grid resolution locally where it is required physically relevant shorter length scale of problem under consideration and adopt coarse mesh size where it is the rest of computational domain, which means that grid inhomogeneity is an essential ingredient in modern large scale numerical simulations.

However, in the problem of wave propagation, the main challenge to use grid inhomogeneity in numerical simulations is the spurious reflection, which is a nonphysical reflection wave occurring when a wave passes through the interface between two regions with grid size inhomogeneity. When wave packet propagating through the numerical interface between domain with different grid sizes, the waves will split into transmitted and reflected components due to the artificial nonphysical interface. Actually, wave propagation in discrete system has been studied since the 17th century and has been described by Brillouin in Ref. [6]. A one-dimensional lattice of a point mass connected by springs has been considered as a model for wave propagation in crystals. Bazănt in $[2,3,7]$ found that when elastic wave pass through the different scale meshes, the spurious reflection may appear at the interface, and the effect of consistent and lumped mass models. The finite element approximations of nonlinear elastic waves were studied in Ref. [21]. In Ref [16], Jiang et al. studied the spurious wave reflection at the interface of different physical properties in finite element wave solution. The optimal reduction for wave propagation problems in the numerical dispersion relationship using two-dimensional elements is discussed in Ref. [13]. Kulkarni et al. [14,15] has talked about this topic in the theory of peridynamic, including the wave propagation in a peridynamic bar with nonuniform discretization and the spurious wave reflection at the interface between peridynamics and finite element regions.

One related work in the multi-scale modeling and computation is the coupling of the molecular dynamics model and the continuum model. An important work in this region is the heterogeneous multiscale method (HMM) developed by $\mathrm{E}$ et al. in Ref. $[9,17,20,27]$, which is based on the widely used domain decomposition method in finite elements and the Cauchy-Born rule. Another interesting method is the bridging scale method (BSM) developed by Liu et al., they developed the mathematical framework of the bridging scale method and use a projection process to couple atomistic and continuum simulations using a bridging scale decomposition [26, 28]. Another approach to deal with the interface in PDEs is local time stepping method, when the space is discretized by adaptive mesh. Osher and Sanders studied the 1-D case of nu- 
merical approximations to nonlinear conservation laws with locally varying time and space grids in Ref. [22]. Berger and Oliger [4] investigated an adaptive refined mesh method for hyperbolic partial differential equation. Tan et al. [25] developed an interpolation and flux correction technique to the interface condition of moving mesh method for hyperbolic system.

In this work, we focus on the development of numerical technique to eliminate the spurious wave reflection at the interface between domains with grid inhomogeneity by the introduction of hierarchical interface conditions (HICs). The source of the spurious reflection could be studied in variational perspective, and then the newly proposed interface condition is introduced with the help of a new auxiliary variable in order to eliminate the source term at the interface produced by the grid inhomogeneity of the approximation in space.

The dynamic of the auxiliary variable in simulation could be studied by using the Padé Via Lanczos (PVL) method. Thus an efficient and applicable interface condition for wave equation has been built. The PVL method is an algorithm for computing the Padé approximation of Laplace-domain transfer functions of large linear networks via a Lanczos process. The PVL algorithm has significantly superior numerical stability, while retaining the same efficiency as algorithms that compute the Padé approximation directly through moment matching such as the asymptotic waveform evaluation (AWE) technique and its derivatives. The study of the theoretical connection between Padé approximation and Lanczos process can be traced back to 1974 [12] in which a matrix interpretation and applications of the continued fraction algorithm is investigated. It has been applied in $[10,11]$ instead of the AWE method [23] to provide an efficient circuit simulation methods due to its excellent numerical stability. The PVL method is also used to study the electromagnetic radiation problems to solve FEM equations with a reduction in total computation time [24]. A comprehensive overview of the Lanczosbased Padé approximation, also known as the PVL method is presented in Ref. [5].

The remaining part of this paper is organized as follows. In Section 2, we introduce our view of the spurious reflection with an auxiliary variable. In Section 3, the PVL method is applied to solve the equation and a convergent theorem is given. Then in Section 4, an implementation of our method on the basic wave equation is given, while we end up our article in Section 5 by conclusion.

\section{The source of spurious reflection and hierarchical interface conditions}

In this section, we first derive the source of spurious reflection due to the inhomogeneity or nonuniform discretization in numerical calculation from variational perspective. An extended Lagrangian functional is developed with the help of an auxiliary variable $w$ in order to eliminate the effects of spurious reflection.

We start our derivation by considering a simple connected region $\Omega$ in $\mathbf{R}^{n}$, which is contained in the configuration manifold of our interested scalar wave equation system. A function space $X$ is defined on $\Omega \times T$ where $T=\{t \mid t \in[0, T]\}$, which is used to describe some physical quantities of the wave equation system. As we have stated in 
previous section, the whole computational domain $\Omega$ is decomposed into two subdomains $\Omega_{1}$ and $\Omega_{2}$, where $\Omega=\Omega_{1} \cup \Omega_{2}$. We discretize domain $\Omega_{1}$, which is the region we need more information, with very fine mesh and domain $\Omega_{2}$ with relatively coarse mesh in order to simulate the grid size inhomogeneity in wave propagation problem. The boundary of $\Omega_{1}$ and $\Omega_{2}$ is $\partial \Omega_{1}$ and $\partial \Omega_{2}$, respectively, and therefore the interface between $\Omega_{1}$ and $\Omega_{2}$ is denoted as $\Gamma=\partial \Omega_{1} \cap \Omega_{2}$.

\subsection{Spurious reflection}

In general wave system, the kinetic energy density functional can be written as $T[u, v]=\left\langle\partial_{t} u, \partial_{t} v\right\rangle / 2$, and we also can define a potential energy functional $V[u, v]=$ $\langle D u, D v\rangle / 2$, where $u, v$ are functions defined on $X,\langle\cdot, \cdot\rangle$ is the inner product, $D$ is a gradient operator in corresponding space. Therefore, the positive kinetic and potential energy density in wave system can be directly written as

$$
T(u)=T[u, u]=\frac{(\partial u / \partial t)^{2}}{2}, \quad V(u)=V[u, u]=\frac{\langle D u, D u\rangle}{2}=\frac{\|D u\|^{2}}{2},
$$

respectively. Therefore, the total kinetic energy and potential energy in $\Omega$ is

$$
\mathcal{T}=\frac{1}{2} \int_{\Omega}\left\|u_{t}\right\|^{2} d \mathbf{x}, \quad \mathcal{V}=\frac{1}{2} \int_{\Omega}\|\nabla u\|^{2} d \mathbf{x} .
$$

Let us denote an elliptic operator $L: X \rightarrow X^{*}$ such that $L=-\Delta$ where $X^{*}$ is the dual space of $X$. The Lagrangian functional $\mathcal{L}$ of the wave equation associated with all these definitions can be written as

$$
\mathcal{L}[u(\mathbf{x}, t)]=T(u)-V(u)=\frac{1}{2}\left\langle u_{t}, u_{t}\right\rangle-\frac{1}{2}\langle D u, D u\rangle .
$$

If there is an additional generalized external force distribution $f \in X^{*}$ is applied to the wave system and suppose that $f$ is compactly supported in the finely discretized subdomain $\Omega_{1} \subseteq \Omega$. $\Omega_{1}$ is the region we are more interested, in which the phenomenon related to source, sink and/or other obstacles are occurred, and therefore more information need to be calculated in this domain with finely discretized mesh. We can rewrite the Lagrangian functional $\mathcal{L}$ in forced system as

$$
\mathcal{L}[u(\mathbf{x}, t)]=T(u)-V(u)-W(u)=\frac{1}{2}\left\langle u_{t}, u_{t}\right\rangle-\frac{1}{2}\langle D u, D u\rangle-\langle f, u\rangle .
$$

The stationary action principle, also known as the principle of least action, says that the dynamical process of the wave system should be the minimizer of action functional $\mathcal{S}$, i.e., the integral of Lagrangian over $\Omega \times T$

$$
u(\mathbf{x}, t)=\underset{\tilde{u}(\mathbf{x}, t) \in X}{\operatorname{argmin}} \mathcal{S}=\underset{\tilde{u}(\mathbf{x}, t) \in X}{\operatorname{argmin}} \int_{\Omega \times T} \mathcal{L}[\tilde{u}(\mathbf{x}, t)] d \mathbf{y},
$$


where $\mathbf{y} \in \Omega \times T$. Taking the variation of action functional with respect to $u$, the weak form of famous Euler-Lagrange (E-L) equation for the wave system, which describe the geodesic flow of the field $u$ as a function of time can be written as

$$
\delta \mathcal{S}=\frac{d}{d \varepsilon} \int_{\Omega \times T} \mathcal{L}[u(\mathbf{x}, t)+\varepsilon v(\mathbf{x}, t)] d \mathbf{y},
$$

where $v$ is a function defined on $X$. Since the system is in stationary state at any time, and therefore the weak form of variation of the action functional can be obtained with the help of integration as following:

$$
\int_{\Omega}\left\langle u_{t t}+L u, v\right\rangle d \mathbf{x}+\int_{\partial \Omega}\langle B u, T v\rangle d \mathbf{x}=\int_{\Omega_{1}}\langle f, v\rangle d \mathbf{x},
$$

where $T$ is the trace operator and $B$ maps from $X$ to $T(X)^{*}$. For instance, in general Euclidean space, the gradient operator is $D=\nabla$, and therefore it can be derived that $L=-\Delta$ and $B u=\nabla_{\mathbf{n}} u$.

In numerical simulation of the wave system, due to the reason that all the solution need to be obtained in discrete system, we need to replace Lagrangian $\mathcal{L}[u]$ by $\mathcal{L}[P u]$, where $P$ is the projector whose range is the space we concern, and usually is a closed invariant space of $L$. However, since we have the grid size inhomogeneity in wave system, i.e., the grid size is larger in $\Omega_{2}$ than that in $\Omega_{1}$, so the project operator $P$ is different in those two domains. In $\Omega_{1}$, the projector could be written as $P_{1}$, where $P_{1}$ projects the function onto a finite dimensional finely discretized finite element space, and in $\Omega_{2}$, the projector could be written as $P \cdot P_{1}$, where $P$ is a relative projector mapping an element in finely discretized space onto a coarsely discretized space. In the following part of the paper, we denote the discretized solution in domain $\Omega_{1}$ as $u$ instead of $P_{1} u$, i.e., omit the operator $P_{1}$ for simplicity. Therefore, the discretized solution in domain $\Omega_{2}$ as $P u$ instead of $P \cdot P_{1} u$.

The Lagrangian in different domains could be written as

$$
\mathcal{L}[u(\mathbf{x}, t)]=\frac{1}{2}\left\langle u_{t}, u_{t}\right\rangle-\frac{1}{2}\langle D u, D u\rangle-\langle f, u\rangle \quad \text { in } \Omega_{1}
$$

and

$$
\mathcal{L}[u(\mathbf{x}, t)]=\frac{1}{2}\left\langle P u_{t}, P u_{t}\right\rangle-\frac{1}{2}\langle D P u, D P u\rangle-\langle f, P u\rangle \quad \text { in } \Omega_{2} .
$$

Hence the new total action functional in numerical sense is

$$
\begin{aligned}
\mathcal{S}^{*}[u ; P]= & \frac{1}{2} \int_{\Omega_{1} \times T}\left(u_{t}^{2}-\langle D u, D u\rangle-\langle f, u\rangle\right) d \mathbf{y} \\
& +\frac{1}{2} \int_{\Omega_{2} \times T}\left(\left(P u_{t}\right)^{2}-\langle D P u, D P u\rangle\right) d \mathbf{y},
\end{aligned}
$$

the weak form of Euler-Lagrange equation of the action functional $\mathcal{S}^{*}$ can be obtained

$$
\int_{\Omega_{1}}\left\langle u_{t t}+L u, v\right\rangle d \mathbf{x}+\int_{\Omega_{2}}\left\langle(P u)_{t t}+L P u, v\right\rangle d \mathbf{x}
$$




$$
+\int_{\partial \Omega_{1}}\langle B u, T v\rangle d \mathbf{x}+\int_{\partial \Omega_{2}}\langle B P u, T v\rangle d \mathbf{x}=\int_{\Omega_{1}}\langle f, v\rangle d \mathbf{x} .
$$

It is worth to note that Eq. (2.7) is the very equation that we solved in numerical simulations. We could also quickly get the corresponding expression under the assumption that the whole domain $\Omega$ is finely discretized using the same uniform mesh as those used in $\Omega_{1}$ that

$$
\int_{\Omega}\left\langle u_{t t}+L u, v\right\rangle d \mathbf{x}+\int_{\partial \Omega}\langle B u, T v\rangle d \mathbf{x}=\int_{\Omega_{1}}\langle f, v\rangle d \mathbf{x}
$$

This is the equation we would like to try our best to approximate using numerical method. Noticing that $\Gamma$ takes different orientation in $\partial \Omega_{1}$ and $\partial \Omega_{2}$, then we could obtain the error of two equations due to the grid size inhomogeneity in $\Omega_{1}$ and $\Omega_{2}$ by subtracting Eq. (2.7) from Eq. (2.8)

$$
\begin{aligned}
& \int_{\Omega_{2}}\left\langle(I-P) u_{t t}+L(I-P) u, v\right\rangle d \mathbf{x}+\int_{\partial \Omega_{2} / \Gamma}\langle B(I-P) u, T v\rangle d \mathbf{x} \\
= & \int_{\Gamma}\left\langle B(I-P) u, T_{\Gamma} v\right\rangle d \mathbf{x},
\end{aligned}
$$

where $T_{\Gamma}$ is the trace operator mapping the function on region $\Omega_{2}$ to its boundary $\Gamma$.

Eq. (2.9) indicates the terms needed to be decreased as possible as we can. It can be found that the first term and the second term is the approximation error, which is unavoidable in model reduction. We denote them with $\operatorname{err}(P, u)$ and we will not try to eliminate this error. The third term in Eq. (2.9) comes from the artificial interface $\Gamma$ between $\Omega_{1}$ and $\Omega_{2}$, therefore that contribution is the main source of the spurious reflection.

\subsection{Hierarchical interface conditions}

In this subsection, we try to eliminate the spurious reflection by proposing hierarchical interface conditions to account the effect of artificial interface between domains with grid inhomogeneity.

From previous subsection, we already found that the third term in Eq. (2.9) is the main source of spurious reflection, so if we could add that term back to our numerical simulation scheme (i.e., Eq. (2.7)), then the main contribution of spurious reflection could be suppressed, and the weak form of Euler-Lagrange equation for actual numerical simulations.

Since $\int_{\Gamma}\left\langle B(P-I) u, T_{\Gamma} v\right\rangle d \mathbf{x}$ is the term acts as a boundary traction force on $\Omega_{1}$, so we propose a new numerical method by adding this traction force to the wave system as an interface condition, i.e., the so-called hierarchical interface condition (HIC) method. In this method, we are solving a surrogate system

$$
\int_{\Omega}\left\langle u_{t t}+L u, v\right\rangle d \mathbf{x}+\int_{\partial \Omega}\langle B u, T v\rangle d \mathbf{x}
$$




$$
=\int_{\Omega}\langle f, v\rangle+\int_{\Gamma}\left\langle B(P-I) u, T_{\Gamma} v\right\rangle d \mathbf{x}
$$

The right-hand side, which play a role of external force, could also be written as

$$
\begin{aligned}
& \int_{\Omega}\langle f, v\rangle+\int_{\Gamma}\left\langle B(P-I) u, T_{\Gamma} v\right\rangle d \mathbf{x} \\
= & \int_{\Omega}\left\langle f+T_{\Gamma}^{T} B(P-I) u, v\right\rangle=\int_{\Omega}\left\langle f_{m}, v\right\rangle d \mathbf{x},
\end{aligned}
$$

where $f_{m}$ is the modified external force. In these hierarchical interface conditions, the additional boundary traction force could also generate error in coarsely discretized domain $\Omega_{2}$. In order to eliminate this error, a new auxiliary variable $w$ is introduced in coarsely discretized domain $\Omega_{2}$ and the extended action functional $\mathcal{S}_{e}$ could be written as

$$
\mathcal{S}_{e}[u, w]=\mathcal{S}^{*}[u]+\frac{1}{2} \int_{\Omega_{2}}\left(w_{t}^{2} d x-\langle D w, D w\rangle\right) d \mathbf{x},
$$

while the boundary condition for variable $w$ on $\Gamma$ is Dirichlet boundary condition

$$
\left.w\right|_{\Gamma}=(P-I) u
$$

and $w$ takes the absorbing boundary condition on $\partial \Omega_{2} / \Gamma$

$$
\left.B w\right|_{\partial \Omega_{2} / \Gamma}=0 .
$$

The weak form of the Euler-Lagrange equation for this newly proposed extended action function $\mathcal{S}_{e}$ can be derived in the same way as what we have done, it can be easily found that the effects of artificial interface, i.e., the spurious wave reflection could be canceled with the help of new variable $w$. Therefore, the new HIC method for wave equation system could be proposed by solving two variable: $u$ for the solution in both $\Omega_{1}$ and $\Omega_{2}$, and $w$ for the auxiliary variable in $\Omega_{2}$. The corresponding evolution equation for $w$ is

$$
\left\{\begin{array}{l}
w_{t t}+L w=0, \\
\left.w\right|_{\Gamma}=(P-I) u, \\
\left.B w\right|_{\partial \Omega_{2} / \Gamma}=0 .
\end{array}\right.
$$

Therefore, the modified external force could also be written as

$$
f=f_{m}-T_{\Gamma}^{T} B w,
$$

which means that the correction can be obtained by extracting the $T_{\Gamma}^{T} B w$ term in Eq. (2.16). The wave equation for $u$ can be written as

$$
u_{t t}+L u=f_{m} .
$$

Finally, the new HIC method, which could suppress the spurious reflection, is performed by numerically solving Eqs. (2.10), (2.11), (2.15), and (2.16). It is worthy to 
note that this method could also be generated to the case that the medium with wave speed inhomogeneity by changing the form of Eq. (2.7) and deriving the similar HIC method with new variable as shown in Eq. (2.15). Since the spurious reflection occurs due to the interface between inhomogeneous grids, the key problem now is how to efficiently simulate the additional dynamical process of auxiliary variable $w$ in domain $\Omega_{2}$.

\section{PVL method for auxiliary variable $w$}

In this section, the PVL method is applied to solve the dynamic evolution of auxiliary variable $w$. We denote a series of finite dimension subspace as $X_{n} \subseteq X$, then the space discretization of $w$ in $X_{n}$ is determined as $w_{n}$, which is a function in $X_{n}$ s.t.

$$
\int_{\Omega_{2}}\left\langle\frac{\partial^{2} w_{n}}{\partial t^{2}}, v_{n}\right\rangle+\left\langle L w_{n}, v_{n}\right\rangle+\left\langle T_{\Gamma}^{T} B(P-I) u, v_{n}\right\rangle d \mathbf{x}=0
$$

for all $v_{n} \in X_{n}$. The key point of the discretization, which depends on the choice of $X_{n}$, is to balance the accuracy and the degree of freedom of this finite dimension subspaces. Here the boundary condition on $\Omega_{2} / \Gamma$ is omitted. In practice, the perfectly matched layer method (PML) or the damping method could be applied in numerical simulations to eliminate the far field of $w$.

To analyse Eq. (3.1), we can equivalently represent the system in the frequency domain instead of the time domain via a Laplace transform

$$
\hat{w}_{n}(\mathbf{x}, s):=\int_{0}^{\infty} w_{n}(\mathbf{x}, t) e^{-s t} \mathrm{~d} t, \quad s \in \mathbf{C}
$$

which leads to

$$
s^{2}\left\langle G \hat{w}_{n}, \hat{v}_{n}\right\rangle-\left\langle L \hat{w}_{n}, \hat{v}_{n}\right\rangle=\left\langle-T_{\Gamma}^{T} B(P-I) \hat{u}, \hat{v}_{n}\right\rangle,
$$

where $G: X \rightarrow X^{*}$ is the operator associated with the Laplace transformation. The Eq. (3.3) solves

$$
\hat{w}_{n}=\left(L-s^{2} G\right)^{-1} Q_{n} T_{\Gamma}^{T} B(P-I) \hat{u},
$$

where $Q_{n}$ is the projector to $L\left(X_{n}\right)^{*}$.

Next, a fixed point $s_{0}$ should be picked according to the $P$ in Section 2 to expand Eq. (3.4). The choice of the point $s_{0}$ should follow three principles: Firstly, $s_{0}^{-2} L-G$ has to be a invertible operator. Then, the frequency of the vector in $\operatorname{Range}(I-P)$ should concentrate on the neighbourhood of $s_{0}$, a quantitative description of which is given in Theorem 3.1 below. It will directly decide the quality of the HIC. And finally, the practical implementation requires that $s_{0}^{-2} L-G$ should be easily computed. Under such consideration, $s_{0}=0$ and $s_{0}=+\infty$ are two most frequently choices, and it serves for most application scenarios. For example, if $L=-\Delta$ and $P$ is the low frequency 
filter, then $s_{0}$ should be $\infty$ since $w$ represents the high frequency wave in $\Omega_{2}$. Denote $R=\left(s_{0}^{-2} L-G\right)^{-1}$ and $A=\left(s_{0}^{-2} L-G\right)^{-1} L$, the expression of $\hat{w}_{n}$ could be written as

$$
\begin{aligned}
\hat{w}_{n} & =\left(L-s^{2} G\right)^{-1} Q_{n} T_{\Gamma}^{T} B(P-I) \hat{u} \\
& =s^{-2}\left[\left(s_{0}^{-2} L-G\right)+\left(s^{-2}-s_{0}^{-2}\right) L\right]^{-1} Q_{n} T_{\Gamma}^{T} B(P-I) \hat{u} \\
& =s^{-2}\left[I-\left(s_{0}^{-2}-s^{-2}\right) A\right]^{-1} R Q_{n} T_{\Gamma}^{T} B(P-I) \hat{u} \\
& =s^{-2} K_{n}\left(s, s_{0}\right) T_{\Gamma}^{T} B(P-I) \hat{u} .
\end{aligned}
$$

\subsection{The Padé approximation}

It can be apparently observed that to what degree $K_{n}\left(s, s_{0}\right)$ is a good approximation of $K\left(s, s_{0}\right)=\left(s^{-2} L-G\right)^{-1}$ numerically is depending on the space discretization. Since the fact that $K_{n}$ could be written as a rational function of $s$, it is natural to consider by using Padé approximation to obtain $K_{n}$ numerically, i.e., we would like to get the Padé approximation of $K(s)$ of order $n$

$$
K_{n}(s)=\frac{a_{n-1} s^{n-1}+\cdots+a_{1} s+a_{0}}{b_{n} s^{n}+b_{n-1} s^{n-1}+\cdots+b_{1} s+1} .
$$

Now we need to find a proper metric to measure our approximation. Noticing that in Eqs. (2.16), (3.5), the trace operator $T_{\Gamma}$ is multiplied on the both side of $K(s)$, then we are aware of that Range $\left(Q_{n} T_{\Gamma}^{T}\right)$ should be a relative small linear space, i.e., $l:=\operatorname{dim}\left(\operatorname{Range}\left(Q_{n} T_{\Gamma}^{T}\right)\right) \sim \mathcal{O}(1)$. For example, in the linear finite element space, $l$ is less than the number of the nodes that are directly connected with $\Gamma$. Therefore, we define $K_{n}$ being the rational function satisfying the following asymptotic condition:

$$
q_{1}^{T} B\left[K_{n}\left(s, s_{0}\right)-K\left(s, s_{0}\right)\right] q_{2} \leq C_{\text {Pade }}\left(\frac{1}{s_{0}}-\frac{1}{s}\right)^{2 n}
$$

for any normalized $q_{1}, q_{2} \in \operatorname{Range}\left(Q_{n} T_{\Gamma}^{T}\right)$. Then we can obtain the convergence theorem of Padé approximation.

Theorem 3.1 (Convergence of $u$ using Padé approximation). If we denote $u^{n}$ being the solution of Eq. (2.17) with the space discretization $K_{n}$ and the following conditions are satisfied:

- For any $q \in \operatorname{Range}\left(T_{\Gamma}^{T}\right)$, we have

$$
\left\|Q_{n} q-q\right\|<C_{\text {proj }} \frac{\|q\|}{n^{\alpha_{1}}} .
$$

This is the condition could be called approximation of the projector.

- The frequency of $(P-I) u$ concentrates on the neighbourhood of $s_{0}$

$$
\left\|\left(L-s^{2} G\right)^{-1} T_{\Gamma}^{T} B(P-I) \hat{u}(s)\right\| \leq C_{\text {freq }} \frac{\|\hat{u}(s)\|}{\left|s-s_{0}\right|^{1 / 2+\alpha_{2}}},
$$

when $\left|s-s_{0}\right|>M$. This condition could be called as frequency concentration. 
Then we have

$$
\begin{aligned}
& \left\|u^{n}-u\right\|_{L^{2}(\Omega)}^{2}(t) \\
& \leq C\left\{n^{-2 \alpha_{1}} \int_{0}^{t}(t-\tau) \exp (t-\tau)\|u\|^{2}(\tau) d \tau\right. \\
& \left.\quad+\left[\frac{1}{\sqrt{n}}\left(\frac{M}{2 \beta^{2}}\right)^{2 n+1}+M^{-\alpha}\right]^{2} \int_{0}^{\infty}\|u\|^{2}(\tau) \exp [2 \beta(t-\tau)] d \tau\right\},
\end{aligned}
$$

where $C$ only depends on $\left\{L, G, C_{\text {freq }}, C_{\text {proj }}\right\}$.

Proof. In region $\Omega$, the following two important conclusions which will be used repeatedly later can be easily obtained:

$$
\begin{aligned}
& \frac{\partial\|v\|_{L^{2}(\Omega)}^{2}}{\partial t}=2 \int_{\Omega}\left\langle v, v_{t}\right\rangle d x \leq\|v\|_{L^{2}(\Omega)}^{2}+\left\|v_{t}\right\|_{L^{2}(\Omega)}^{2}, \\
& \|v\|_{L^{2}(\Omega)}^{2}(t) \leq \exp (t)\|v\|_{L^{2}(\Omega)}^{2}(0)+\int_{0}^{t} \exp (t-\tau)\left\|v_{t}\right\|_{L^{2}(\Omega)}^{2}(\tau) d \tau .
\end{aligned}
$$

By using Eq. (2.16), we get that $u^{n}$ satisfies

$$
\begin{aligned}
& \int_{\Omega}\left(u_{t t}^{n}-L u^{n}+T^{T} B u^{n}\right) d \mathbf{x} \\
= & \int_{\Omega}\left(f+T_{\Gamma}^{T} B\left[w_{n}-(P-I) u^{n}\right]\right) d \mathbf{x}+\operatorname{err}\left(P, u^{n}\right) .
\end{aligned}
$$

Therefore, we get the evolution equation of the approximation error that

$$
\begin{aligned}
& \int_{\Omega}\left(e_{t t}^{n}-L e^{n}+T^{T} B e^{n}+T_{\Gamma}^{T} B(P-I) e^{n}\right) d \mathbf{x} \\
= & \int_{\Omega} T_{\Gamma}^{T} B\left(w-w_{n}\right) d \mathbf{x}+\operatorname{err}\left(P, e^{n}\right),
\end{aligned}
$$

where $e^{n}=u-u^{n}$. If we denote $\delta f=T_{\Gamma}^{T} B\left(w-w_{n}\right)$, by Eq. (3.5) we have

$$
\begin{aligned}
& Q_{n} \hat{\delta f}=Q_{n} T_{\Gamma}^{T} B\left(\hat{w}-\hat{w}_{n}\right) \\
= & s^{-2} Q_{n} T_{\Gamma}^{T} B\left[\left(K-K_{n}\right) Q_{n}+K\left(I-Q_{n}\right)\right] T_{\Gamma}^{T} B(P-I) \hat{u} \\
& -s^{-2} Q_{n} T_{\Gamma}^{T} B K_{n} Q_{n} T_{\Gamma}^{T} B(P-I) \hat{e}_{n} .
\end{aligned}
$$

Thus according to the inverse Laplace transformation we can acquire that

$$
\begin{aligned}
& Q_{n} \delta f=L^{-1}\left\{Q_{n} \hat{\delta f}\right\} \\
= & \frac{1}{2 \pi i} \int_{\beta-i \infty}^{\beta+i \infty} s^{-2} Q_{n} T_{\Gamma}^{T} B\left(K-K_{n}\right) Q_{n} T_{\Gamma}^{T} B(P-I) \hat{u} \cdot \exp (s t) d s
\end{aligned}
$$




$$
\begin{aligned}
& \quad+\frac{1}{2 \pi i} \int_{\beta-i \infty}^{\beta+i \infty} s^{-2} Q_{n} T_{\Gamma}^{T} B K\left(I-Q_{n}\right) T_{\Gamma}^{T} B(P-I) \hat{u} \cdot \exp (s t) d s \\
& \quad-\frac{1}{2 \pi i} \int_{\beta-i \infty}^{\beta+i \infty} s^{-2} Q_{n} T_{\Gamma}^{T} B K_{n} Q_{n} T_{\Gamma}^{T} B(P-I) \hat{e}_{n} \cdot \exp (s t) d s \\
& =r_{1}+r_{2}-r_{3},
\end{aligned}
$$

where $\beta=\mathcal{R} e\left(s_{0}\right), r_{1}, r_{2}$ and $r_{3}$ are the three sequential components in the above equation. In the following derivation, we consider these three terms individually.

For the first term $r_{1}$, we can separate the integral into two parts

$$
\begin{aligned}
& \frac{1}{2 \pi i} \int_{\beta-i \infty}^{\beta+i \infty} \frac{1}{s^{2}} Q_{n} T_{\Gamma}^{T} B\left(K-K_{n}\right) Q_{n} T_{\Gamma}^{T} B(P-I) \hat{u} \cdot \exp (s t) d s \\
& =\frac{1}{2 \pi i} \int_{s_{0}-i M}^{s_{0}+i M} \frac{1}{s^{2}} Q_{n} T_{\Gamma}^{T} B\left(K-K_{n}\right) Q_{n} T_{\Gamma}^{T} B(P-I) \hat{u} \cdot \exp (s t) d s \\
& \quad+\int_{\left|s-s_{0}\right|>M} \frac{1}{s^{2}} Q_{n} T_{\Gamma}^{T} B\left(K-K_{n}\right) Q_{n} T_{\Gamma}^{T} B(P-I) \hat{u} \cdot \exp (s t) d s .
\end{aligned}
$$

The estimation of the first part in $r_{1}$ can be obtained with the help of Padé approximation

$$
\begin{aligned}
& \left\|\frac{1}{2 \pi i} \int_{s_{0}-i M}^{s_{0}+i M} s^{-2} Q_{n} T_{\Gamma}^{T} B\left(K-K_{n}\right) Q_{n} T_{\Gamma}^{T} B(P-I) \hat{u} \cdot \exp (s t) d s\right\| \\
\leq & \frac{C_{\text {Pade }}}{2 \pi}\left|\int_{s_{0}-i M}^{s_{0}+i M}\left(\frac{1}{s_{0}}-\frac{1}{s}\right)^{2 n} \frac{d s}{s^{2}}\right|^{\frac{1}{2}}\left[\int_{s_{0}-i M}^{s_{0}+i M}\left\|T_{\Gamma}^{T} B(P-I) \hat{u}\right\|^{2} \exp (s t) d s\right]^{\frac{1}{2}} \\
\leq & \frac{C_{\text {Pade }}}{2 \pi}\left|\int_{1 /\left(s_{0}-i M\right)}^{1 /\left(s_{0}+i M\right)}\left(\frac{1}{s_{0}}-w\right)^{2 n} d w\right|^{\frac{1}{2}}\left[\int\left\|T_{\Gamma}^{T} B(P-I) \hat{u}\right\|^{2} \exp (2 \beta t) d s\right]^{\frac{1}{2}} \\
\leq & \frac{C_{\text {Pade }} C_{\text {trace }}\|B\|}{\sqrt{2 n+1} \pi}\left(\frac{\phi}{2 \beta}\right)^{n+\frac{1}{2}}\left[\int_{0}^{\infty}\|u\|^{2}(\tau) \exp [2 \beta(t-\tau)] d \tau\right]^{\frac{1}{2}},
\end{aligned}
$$

where we use the relation

$$
\phi=\frac{1}{2}\left|\arg \left(\frac{s_{0}+i M}{s_{0}-i M}\right)\right| \leq \frac{M}{\beta}
$$

and therefore we can take $M<\sqrt{2} \beta^{2}$ to ensure that $\left|\frac{\phi}{2 \beta}\right|<1$.

The estimation of the second part in $r_{1}$ can be made by using the condition of frequency concentration as following:

$$
\left\|\frac{1}{2 \pi i} \int_{\left|s-s_{0}\right|>M} s^{-2} Q_{n} T_{\Gamma}^{T} B\left(K-K_{n}\right) Q_{n} T_{\Gamma}^{T} B(P-I) \hat{u} \cdot \exp (s t) d s\right\|
$$




$$
\begin{aligned}
& \leq \frac{C_{\text {freq }}}{\pi} \int_{\left|s-s_{0}\right|>M} \frac{1}{|s|^{2}\left|s-s_{0}\right|^{\frac{1}{2}+\alpha_{2}}}\|\hat{u}\|(s) \exp (s t) d s \\
& \leq \frac{C_{\text {freq }}}{\pi}\left(\int_{\left|s-s_{0}\right|>M} \frac{1}{|s|^{4}\left|s-s_{0}\right|^{1+2 \alpha_{2}}}\right)^{\frac{1}{2}}\left(\int_{\left|s-s_{0}\right|>M}\|\hat{u}\|^{2}(s) \cdot \exp (2 s t) d s\right)^{\frac{1}{2}} \\
& \leq \frac{C_{\text {freq }}}{\sqrt{\alpha_{2}} \beta^{2} \pi} M^{-\alpha_{2}}\left[\int_{0}^{\infty}\|u\|^{2}(\tau) \exp [2 \beta(t-\tau)] d \tau\right]^{\frac{1}{2}}
\end{aligned}
$$

For the second term $r_{2}$, we first suppose $r$ be the solution to the following equation:

$$
\left\{\begin{array}{l}
r_{t t}-L r=-\left(I-Q_{n}\right) T_{\Gamma}^{T} B(P-I) u \text { in } \Omega_{2} \\
\left.B r\right|_{\partial \Omega_{2}}=0 \\
\left.r\right|_{t=0}=\left.r_{t}\right|_{t=0}=0
\end{array}\right.
$$

Then it can be obtained that $r_{2}=Q_{n} T_{\Gamma}^{T} B r$. We then define a new functional as following:

$$
\mathcal{H}_{1}\left(\Omega_{2}, r, r_{t}, t\right)=\frac{1}{2} \int_{\Omega_{2}} r_{t}^{2}+\langle L r, r\rangle d \mathbf{x} .
$$

It can be acquired that

$$
\begin{aligned}
\frac{\partial \mathcal{H}_{1}\left(\Omega_{2}, r, r_{t}, t\right)}{\partial t} & =\frac{1}{2} \int_{\Omega_{2}} 2\left\langle r_{t t}, r_{t}\right\rangle+\left\langle L r_{t}, r\right\rangle+\left\langle r_{t}, L r\right\rangle d \mathbf{x} \\
& =\int_{\Omega_{2}}\left\langle r_{t t}+L r, r_{t}\right\rangle d \mathbf{x}-\int_{\partial \Omega_{2}}\left\langle B r, T r_{t}\right\rangle d \mathbf{x} .
\end{aligned}
$$

By using the relation in (3.20), Eq. (3.22) can be written as

$$
\begin{aligned}
\frac{\partial \mathcal{H}_{1}\left(\Omega_{2}, r, r_{t}, t\right)}{\partial t} & =-\int_{\Omega_{2}}\left\langle\left(I-Q_{n}\right) T_{\Gamma}^{T} B(P-I) u, r_{t}\right\rangle d \mathbf{x} \\
& \leq\left\|\left(I-Q_{n}\right) T_{\Gamma}^{T} B(P-I) u\right\| \cdot\left\|r_{t}\right\| \\
& \leq \frac{1}{2}\left\|\left(I-Q_{n}\right) T_{\Gamma}^{T} B(P-I) u\right\|^{2}+\frac{1}{2}\left\|r_{t}\right\|^{2} \\
& \leq \frac{C_{\text {proj }}^{2} C_{\text {trace }}^{2}\|B\|^{2}}{2 n^{2 \alpha_{1}}}\|u\|^{2}+\mathcal{H}_{1}\left(\Omega_{2}, r, r_{t}, t\right) .
\end{aligned}
$$

Therefore, we have

$$
\mathcal{H}_{1}\left(\Omega_{2}, r, r_{t}, t\right) \leq \frac{C_{\text {proj }}^{2} C_{\text {trace }}^{2}\|B\|^{2}}{2 n^{2 \alpha_{1}}} \int_{0}^{t} \exp (t-\tau)\|u\|^{2}(\tau) d \tau .
$$

From the initial conditions of $r$ and $r_{t}$, we can easily get $\mathcal{H}_{1}\left(\Omega_{2}, r, r_{t}, 0\right)=0$. By Eq. (3.12)

$$
\left\|r_{2}\right\|(t) \leq \frac{C_{\text {proj }}^{2} C_{\text {trace }}^{2}\|B\|^{2}}{2 n^{\alpha_{1}}}\left(\int_{0}^{t}(t-\tau) \exp (t-\tau)\|u\|^{2}(\tau) d \tau\right)^{\frac{1}{2}} .
$$


For the third term $r_{3}$, the method used to get the estimation is similar to those for the second term. We suppose that $y \in X_{n}$ is the solution of the following ordinary differential equation:

$$
\left\{\begin{array}{l}
y_{t t}-L y=-Q_{n} T_{\Gamma}^{T} B(P-I) e_{n} \text { in } \Omega_{2} \\
\left.B y\right|_{\partial \Omega_{2}}=0 \\
\left.y\right|_{t=0}=\left.y_{t}\right|_{t=0}=0
\end{array}\right.
$$

Then we have $r_{3}=Q_{n} T_{\Gamma}^{T} B y$. The following estimation can be obtained that:

$$
\left\|r_{3}\right\|(t) \leq C_{\text {trace }}^{2}\|B\|^{2}\left(\int_{0}^{t}(t-\tau) \exp (t-\tau)\left\|e_{n}\right\|^{2}(\tau) d \tau\right)^{\frac{1}{2}} .
$$

In conclusion, we can get the estimation of $\|\delta f\|$ based on the obtained estimations of three components as following:

$$
\begin{aligned}
\|\delta f\| \leq & C_{\text {proj }} \frac{\|\delta f\|}{n^{\alpha_{1}}}+C_{1}\left(\int_{0}^{t}(t-\tau) \exp (t-\tau)\left\|e_{n}\right\|^{2}(\tau) d \tau\right)^{\frac{1}{2}} \\
& +\frac{C_{2}}{n^{\alpha_{1}}}\left(\int_{0}^{t}(t-\tau) \exp (t-\tau)\|u\|^{2}(\tau) d \tau\right)^{\frac{1}{2}} \\
& +C_{3}\left[\frac{1}{\sqrt{n}}\left(\frac{\phi}{2 \beta}\right)^{2 n+1}+M^{-\alpha}\right]\left[\int_{0}^{\infty}\|u\|^{2}(\tau) \exp [2 \beta(t-\tau)] d \tau\right]^{\frac{1}{2}} .
\end{aligned}
$$

Now we denote that

$$
\mathcal{H}_{2}\left(\Omega, e^{n}, e_{t}^{n}, t\right)=\frac{1}{2}\left[\int_{\Omega}\left(e_{t}^{n}\right)^{2} d \mathbf{x}-\int_{\Omega_{1}}\left\langle L e^{n}, e^{n}\right\rangle d \mathbf{x}-\int_{\Omega_{2}}\left\langle L P e^{n}, P e^{n}\right\rangle d \mathbf{x}\right]
$$

and substitute that definition into Eq. (3.14)

$$
\begin{aligned}
\frac{\partial \mathcal{H}_{2}}{\partial t} & =\int_{\Omega}\left\langle\delta f, e_{t}^{n}\right\rangle d \mathbf{x} \leq \frac{1}{2}\|\delta f\|^{2}+\frac{1}{2}\left\|e_{t}^{n}\right\|_{L^{2}(\Omega)}^{2} \\
& \leq \frac{1}{2}\|\delta f\|^{2}+\frac{1}{2} \mathcal{H}_{2}\left(\Omega, e^{n}, e_{t}^{n}, t\right) .
\end{aligned}
$$

Therefore, we have

$$
\mathcal{H}_{2}\left(\Omega, e^{n}, e_{t}^{n}, t\right) \leq \frac{1}{2} \int_{0}^{t} \exp (t-\tau)\|\delta f\|^{2}(\tau) d \tau .
$$

Due to fact that $e^{n}$ has a homogeneous initial value, then by using the relation of Eq. (3.12), we get that

$$
\left\|e^{n}\right\|_{L^{2}(\Omega)}^{2}(t) \leq \frac{1}{2} \int_{0}^{t}(t-\tau) \exp (t-\tau)\|\delta f\|^{2}(\tau) d \tau
$$




$$
\begin{aligned}
& \leq C_{1}^{2} \int_{0}^{t}(t-\tau) \exp (t-\tau)\left\|e_{n}\right\|^{2}(\tau) d \tau+\frac{C_{2}^{2}}{n^{2 \alpha_{1}}} \int_{0}^{t}(t-\tau) \exp (t-\tau)\|u\|^{2}(\tau) d \tau \\
& \quad+C_{3}^{2}\left[\frac{1}{\sqrt{n}}\left(\frac{\phi}{2 \beta}\right)^{2 n+1}+M^{-\alpha}\right]^{2} \int_{0}^{\infty}\|u\|^{2}(\tau) \exp [2 \beta(t-\tau)] d \tau
\end{aligned}
$$

where a constant $3 n^{\alpha_{1}} /\left(n^{\alpha_{1}}-C_{\text {proj }}\right)$ is omitted.

This conclusion tells that the norm of error of $u_{n}$ is bounded at finite time which ensure the convergence of the solution by using Padé approximation.

\subsection{Krylov subspaces and the Lanczos process}

In order to get the approximation $K_{n}\left(s, s_{0}\right)$ as shown in Eq. (3.5), it still remains a question that how to find the $Q_{n}$ efficiently when the Padé approximation is applied. Here we use the PVL method. The basic idea follows the method proposed in Ref. [1].

In Lanczos process we build an orthogonal basis of the Krylov subspace of a linear operator. The Krylov subspace is a subspace spanned by a sequence of vectors generated by a given linear operator, the left vectors $V$ and the right vectors $W$ as follows:

$$
\begin{aligned}
& \mathcal{K}(A, W, n)=\operatorname{Span}\left\{r \mid r=A^{k} w, w \in W, 0 \leq k \leq n-1\right\}, \\
& \mathcal{K}\left(A^{T}, V, n\right)=\operatorname{Span}\left\{r \mid r=v A^{k}, v \in V, 0 \leq k \leq n-1\right\} .
\end{aligned}
$$

Assuming that $T_{n}$ is the tridiagonal matrix generated by the Lanczos process stopped at the $n$ step, and $W_{n}$ and $V_{n}$ is the orthogonal basis of $\mathcal{K}(A, W, n)$ and $\mathcal{K}\left(A^{T}, V, n\right)$, we have the following lemma.

Lemma 3.1. For any $v \in V$ and $w \in W$, we have

$$
v^{T} A^{k} w=v^{T} V_{n} T_{n}^{k} W_{n}^{T} w
$$

for $k \leq 2 n-l_{1}-l_{2}$, where $l_{1}=\operatorname{dim}(\operatorname{Range}(V)), l_{2}=\operatorname{dim}(\operatorname{Range}(W))$. Thus we have

$$
v^{T}\left[(I-\lambda A)^{-1}-\left(I-\lambda V_{n} T_{n} W_{n}^{T}\right)^{-1}\right] w=\mathcal{O}\left(\lambda^{2 n-l_{1}-l_{2}}\right) .
$$

Proof. Write the elements in $T_{n}^{k}$ explicitly

$$
\left(T_{n}^{k}\right)_{i j}=\sum\left(T_{n}\right)_{m_{0} m_{1}}\left(T_{n}\right)_{m_{1} m_{2}} \cdots\left(T_{n}\right)_{m_{k-1} m_{k}},
$$

where $m$ runs through all index chain starts with $i$ end with $j$. But since $T_{n}$ is an tridiagonal matrix, we know that $m$ jumps less than 1 in every step. Therefore,

$$
\max _{0 \leq p \leq k} m_{p} \leq \frac{i+j+k}{2}, \quad \min _{0 \leq p \leq k} m_{p} \geq \frac{i+j-k}{2} .
$$


Since only the first $l_{2}$ elements of $v^{T} V_{n}$ and the first $l_{1}$ elements of $W_{n}^{T} w$ are not zero, the expansion of $v^{T} V_{n} T_{n}^{k} W_{n}^{T} w$ only has the elements in the $\frac{l+k}{2} \times \frac{l+k}{2}$ leading principal submatrix of $T_{n}$, which proofs the conclusion through

$$
\begin{aligned}
& v^{T}\left[(I-\lambda A)^{-1}-\left(I-\lambda V_{n} T_{n} W_{n}^{T}\right)^{-1}\right] w \\
= & \sum_{k>2 n-l_{1}-l_{2}} \lambda^{k} v^{T}\left[A^{k}-V_{n} T_{n}^{k} W_{n}^{T}\right] w=\mathcal{O}\left(\lambda^{2 n-l_{1}-l_{2}}\right) .
\end{aligned}
$$

The proof is complete.

Hence, we could find the Padé approximation of $K\left(s, s_{0}\right)=\left[I-\left(s^{-2}-s_{0}^{-2}\right) A\right]^{-1} R$ through Lanczos process.

\section{Numerical implementation and simulations}

In this section, we presents some $n$ will give the example of the numerical implementation of our method for the wave equation in non-uniform grids. Let us recall the basic wave equation

$$
\begin{cases}u_{t t}=\Delta u+f & \text { in } \Omega, \\ u(\mathbf{x}, 0)=\varphi(\mathbf{x}) & \text { in } \Omega, \\ u_{t}(\mathbf{x}, 0)=\psi(\mathbf{x}) & \text { in } \Omega\end{cases}
$$

with Cauchy value $\varphi$ and $\psi$ and external source $f$.

Here we denote $\Omega=\Omega_{1} \cup \Omega_{2}$ is a finite dimensional bounded region in which the numerical solution needs to be calculated. $\Omega_{1}$ is the region where most interested dynamic phenomenon occurs, of course $\Omega_{1}$ is the region which needs to be finely discretized in numerically scheme, and the support of external force $f$ is in this finely discretized domain, i.e. $\operatorname{supp}\{f\} \subset \Omega_{1} . \Omega_{2}$ is the rest domain and do not need very fine mesh to obtain numerical results. In our numerical simulations, we suppose a fine uniform grid is generated with grid size $h_{1}$ in $\Omega_{1}$, and a coarse grid is generated in $\Omega_{2}$ with grid size $h_{2}$. The projector $P$ in Section 2 is constructed based on the ratio of the grid size between two regions, i.e., the inhomogeneity ratio $r:=h_{2} / h_{1}>1$.

Let us define $B=\left(\psi_{1}(x), \psi_{2}(x), \cdots, \psi_{n}(x)\right)$ where $\psi_{i}(x)$ is the $i$-th basis function in finite element space, and $\Lambda$ be an interpolation operator. Taking

$$
u(x, k \Delta t)=\sum_{i=1}^{n} \vec{u}_{i}^{k} \psi_{i}(x)=B \vec{u}^{k},
$$

where $\vec{u}^{k} \in \mathbf{R}^{n}$, with a simple application of the central differential scheme, Eq. (4.1) becomes

$$
\left\{\begin{array}{l}
\frac{1}{\Delta t^{2}}\left(\vec{u}^{k+1}+\vec{u}^{k-1}-2 \vec{u}^{k}\right)=\Lambda \Delta B \vec{u}^{k}+\Lambda f, \\
\vec{u}^{0}=\Lambda \varphi \\
\frac{1}{\Delta t}\left(\vec{u}^{1}-\vec{u}^{0}\right)=\Lambda \psi .
\end{array}\right.
$$


It leads to

$$
\left\{\begin{array}{l}
\vec{u}^{k+1}=\left(\Delta t^{2} \Lambda \Delta B+2 I\right) \vec{u}^{k}-\vec{u}^{k-1}+\Delta t^{2} \Lambda f \\
\vec{u}^{1}=\Lambda \varphi+\Delta t \Lambda \psi \\
\vec{u}^{0}=\Lambda \varphi
\end{array}\right.
$$

which we call the Traditional Galerkin Method (TGM).

In the numerical implementation of HIC in wave equation, there is a parameter $s_{0}$ need to be determined in Eq. (3.5). In practical, a coarse grid can be approximately considered to be a high frequency filter

$$
P f=\int_{\omega} 1_{|\omega|<A} \hat{f}(\omega) \exp (i \omega t) d \omega
$$

Therefore, a nature choice of $s_{0}$ is $s_{0}=\infty$, which leads to

$$
K\left(s, s_{0}\right)=\left(\frac{1}{s^{2}} L-G\right)^{-1}=-\left[\sum_{l=0}^{+\infty} \frac{1}{s^{2 l}}\left(G^{-1} L\right)^{l}\right] G^{-1}
$$

For sake of simplification, we take the test function space the same as the finite element space, i.e., Range $(B)=\operatorname{Range}(\Lambda)$, in this case $L$ is symmetric and $G=I$. Therefore, the Lanczos process proposed in Section 3.2 becomes an Arnoldi process.

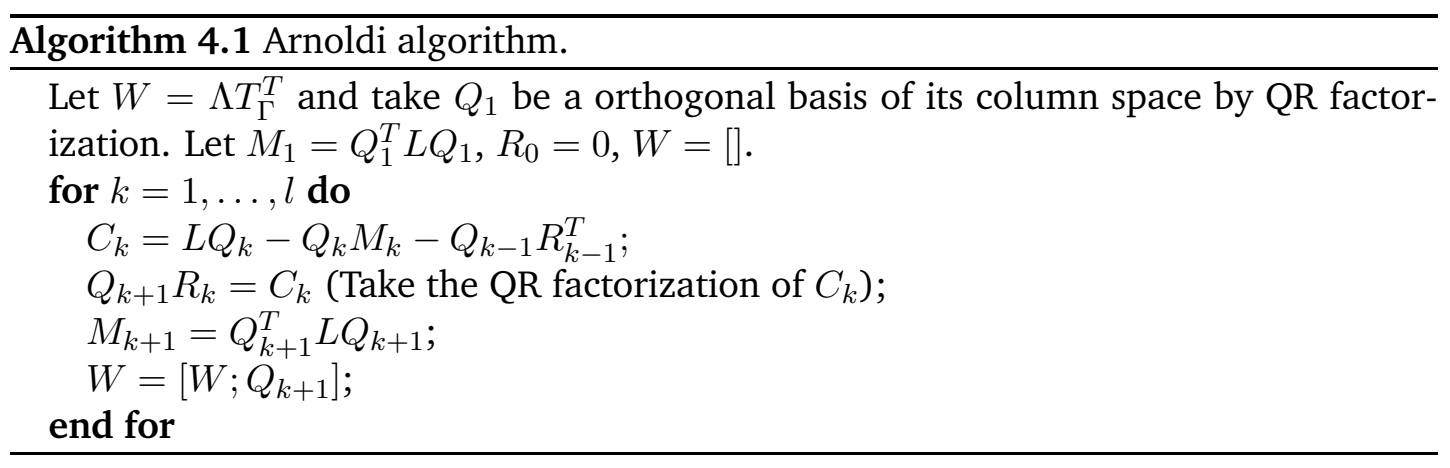

Adding up the auxiliary variable $W \vec{v}^{k}$ and its interpolation operator $\Upsilon$, we have

$$
\begin{aligned}
& u(x, k \Delta t)=B(x) \vec{u}^{k}+W(x) \vec{v}^{k} \\
& \Delta u(x, k \Delta t)=\Lambda \Delta B(x) \vec{u}^{k}+\Upsilon \Delta W(x) \vec{v}^{k}
\end{aligned}
$$

To simulate the absorbing boundary condition of $W \vec{v}^{k}$, a simple PML with $\lambda=\lambda_{\max }\left(\frac{h}{H}\right)^{0}$ is added. Later in the numerical simulation it will be illustrated that the implementation of the absorbing boundary condition is robust. Therefore, the numerical scheme 
shown in Eq. (4.4) becomes

$$
\left\{\begin{array}{l}
\vec{u}^{k+1}=\left(\Delta t^{2} \Lambda \Delta B+2 I\right) \vec{u}^{k}+\Lambda \Delta W \vec{v}^{k}-\vec{u}^{k-1}+\Delta t^{2} \Lambda f \\
\vec{v}^{k+1}=\left(\Delta t^{2} \Upsilon \Delta W+2 I\right) \vec{v}^{k}+\Upsilon \Delta B \vec{u}^{k}-\vec{v}^{k-1}-\Delta t \lambda\left(\vec{v}^{k}-\vec{v}^{k-1}\right), \\
\vec{u}^{1}=\Lambda \varphi+\Delta t \Lambda \psi, \\
\vec{u}^{0}=\Lambda \varphi \\
\vec{v}^{1}=0 \\
\vec{v}^{0}=0 .
\end{array}\right.
$$

That is the numerical implementation of HIC method in wave equation with the help of new variable $\vec{v}^{k}$.

\subsection{One dimensional example}

First we consider the numerical implementation and results in 1-D example. The computational domain $\Omega=[0,10]$ is shown in Fig. 2 . The finely discretized domain is the left half of $\Omega$, i.e., $\Omega_{1}=[0,5]$ with $\Delta x=0.01$ and the coarsely discretized domain is the right half of $\Omega$, i.e., $\Omega_{2}=[5,10]$ with $\Delta x=0.16$. The inhomogeneity ratio in this 1 -D example is $r=16$ and the Courant-Friedrichs-Lewy (CFL) ratio is 0.99. A simple illustration of the FEM space with $r=3$ are given in Fig. 1 .

The initial value $\phi(x)$ and $\psi(x)$ and the external force $f(x)$ are given as following:

$$
\left\{\begin{array}{l}
\varphi(x)=0 \\
\psi(x)=0 \\
f(x)=[\sin (2 t)+0.3 \sin (48 t)] \delta(x) .
\end{array}\right.
$$

It can be found that in this example, the value of $u$ and the gradient of $u$ with respect to time at $t=0$ are 0 and all the waves are stimulated by external force, which is a point

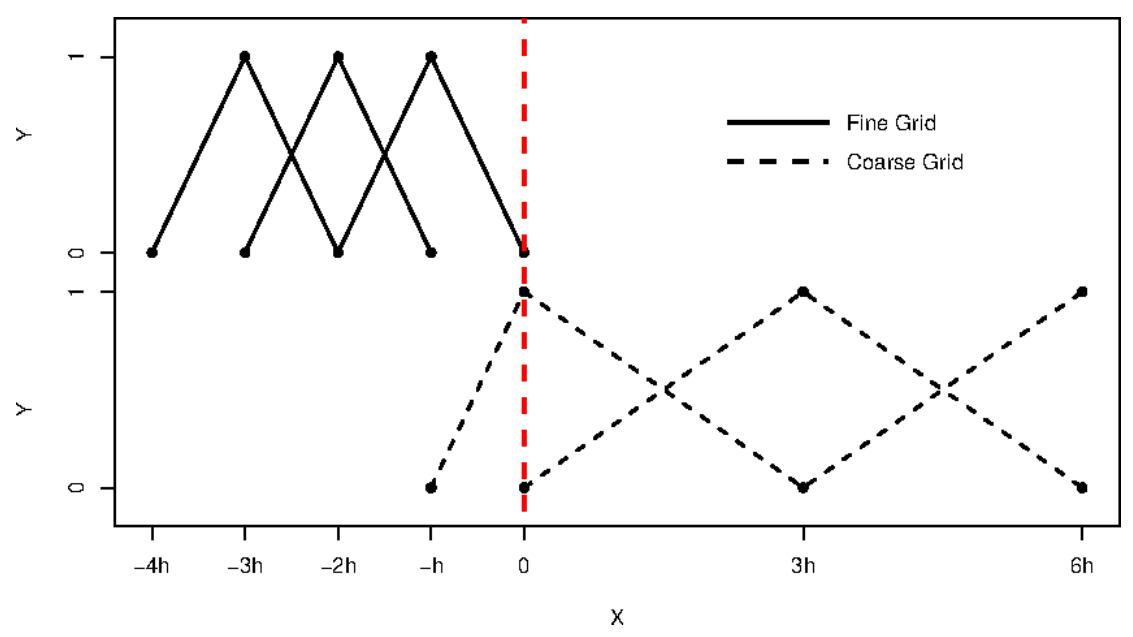

Figure 1: The FEM space in one dimensional case with $r=3$. 

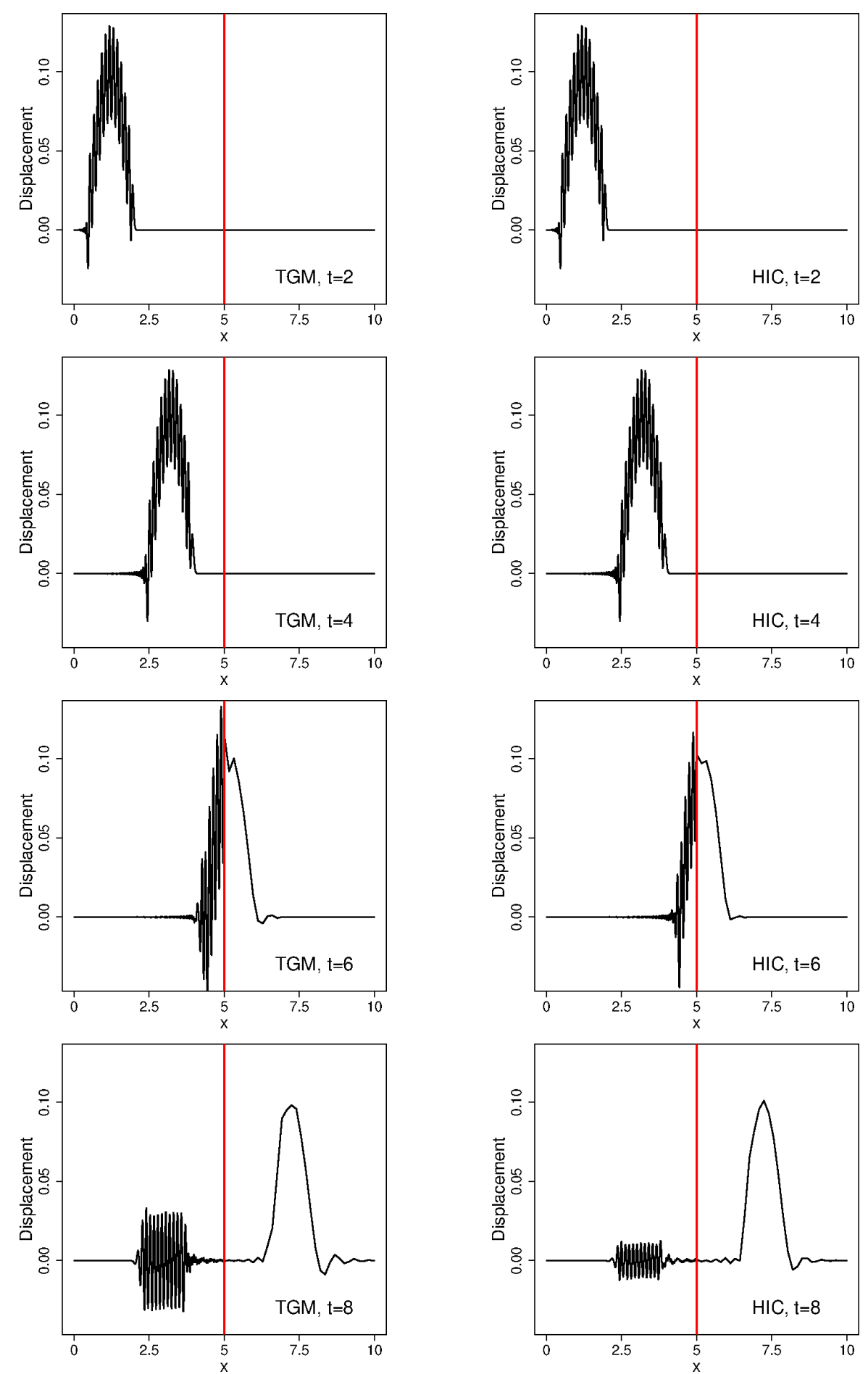

Figure 2: Results of 1-D wave equation at various time. From the top to the bottom is the evolution of time. The first column corresponds to the TGM while the second column to the HIC method. The red halving line is the interface between $\Omega_{1}$ and $\Omega_{2}$. 
source at $x=0$. The stimulated $u$ consists two waves with different frequencies, one is a low frequency wave with $\omega=1 / \pi$ and another is a high frequency wave with $\omega=24 / \pi$. Apparently, the high frequency component can not go through the interface and the spurious reflection occurs.

The results of the numerical simulations for both methods, i.e. TGM and HIC, are shown in the left panel and the right panel of Fig. 2, respectively. Since there are mesh size inhomogeneity in numerical discretization, it can be clearly observed that the high frequency wave can not go through the interface between two discretized region $\Omega_{1}$ and $\Omega_{2}$ as shown in the third and the fourth row in Figs. 2. Since there are no extra process to deal with the interface effect in TGM, we can find that when TGM is applied to solve this 1-D wave equation, there are obvious high frequency spurious reflections as shown in the left panel in Fig. 2 when $t$ is greater than 5 . However, when the method of HIC is applied in numerical simulations, the results in the right panel of Fig. 2 clearly demonstrate that this newly proposed interface condition with the help of auxiliary variable $w$ could effectively reduce the magnitude of the spurious reflection.

In order to compare the results between two methods and to demonstrate the effectiveness of HIC, we also calculate a reference solution for this 1-D example. The reference solution is obtained based on a finely uniform discretized mesh. The finely discretized mesh is generated in the whole domain $\Omega$ instead of only in $\Omega_{1}$. We first perform two different simulations to get the solutions stimulated solely by the low frequency external point source and the high frequency external point source on the fine mesh separately, and then the so-called reference solution could be constructed based on previously obtained two solutions. On domain $\Omega_{1}$, the reference solution

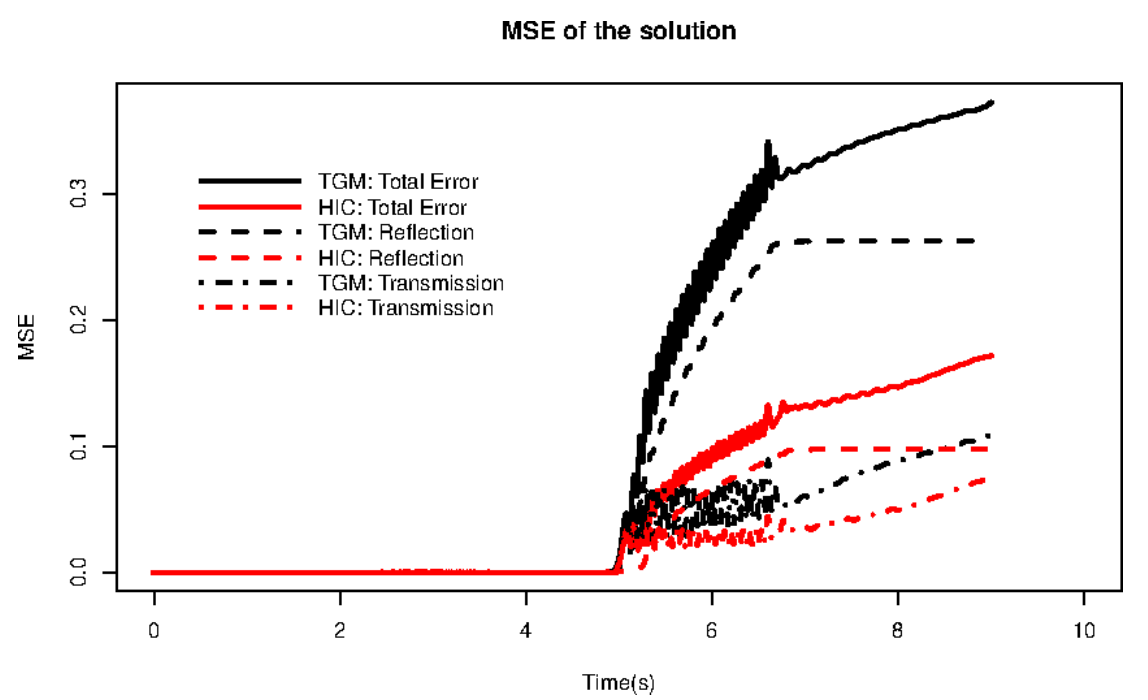

Figure 3: The relative mean square errors at different time for TGM and HIC. The Reflection error is the error calculated in Region with fine grid size, in order to demonstrate the error induced by spurious reflection. The Transmission error is calculated in Region with coarse grid size, in order to demonstrate the error due to the aliasing when high frequency component pass through the interface. 
is the summation of the two waves stimulated by both low frequency and high frequency point source, and on domain $\Omega_{2}$, the reference solution is just equivalent to the wave stimulated by low frequency point source, however, this stimulated wave need to be projected to coarsely discretized mesh in order to compare the results with those obtained in TGM and HIC in domain $\Omega_{2}$. In fact, this reference solution is obtained based on the assumption that the interface between inhomogeneous mesh is a perfect interface which can totally absorb the high frequency components.

The results of the relative mean square error (rMSE) of these two methods comparing with the reference solution is plotted in Fig. 3. The joggling line in the pictures corresponds to the moment when the wave goes though the interface, which is exactly when the error of the HIC method differs with TGM. We also demonstrated the two component of rMSE in fine region $\Omega_{1}$ (TGM:Reflection and HIC:Reflection, respectively) and coarse region $\Omega_{2}$ (TGM:Transmission and HIC:Transmission, respectively) in order the shown the error due to the spurious reflection (in $\Omega_{1}$ ) and transmission aliasing (in $\Omega_{2}$ ). The comparison of the rMSE in Fig. 3 clearly demonstrate that our proposed HIC could effectively reduce the spurious reflection.

\subsection{Two dimensional examples}

In this subsection, we perform two different numerical simulations in 2-D with two different external applied forces. In both simulations, the numerical results are obtain in a rectangle region that $\Omega=[0,10] \times[0,10]$, in which the finely discretized domain is the left half of $\Omega$, i.e., $\Omega_{1}=[0,5] \times[0,10]$ with $\Delta x=5 e-2$ and the coarsely discretized domain is the right half of $\Omega$, i.e., $\Omega_{2}=[5,10] \times[0,10]$ with $\Delta x=5 e-1$. The inhomogeneity ratio between $\Omega_{1}$ and $\Omega_{2}$ is $r=10$ and the Courant-FriedrichsLewy(CFL) ratio is 0.1 . The following two kinds of external force are applied to the wave equation:

$$
\left\{\begin{array}{l}
f_{1}(x, y)=[3 \cos (4 t)+2 \cos (12 t)] \delta\left(x-\frac{10}{3}, y-5\right) \\
f_{2}(x, y)=3 \cos (4 t) \delta\left(x-\frac{40}{9}, y-6\right)+2 \cos (16 t) \delta\left(x-\frac{40}{9}, y-4\right) .
\end{array}\right.
$$

In the first case with external force $f_{1}(x, y)$, both high frequency wave $(\omega=6 / \pi)$ and low frequency wave $(\omega=2 / \pi)$ are stimulated at the same point source at $(x=$ $10 / 3, y=5)$. In the second case with external force $f_{2}(x, y)$, the high frequency wave $(\omega=8 / \pi)$ and low frequency wave $(\omega=2 / \pi)$ is stimulated at the different point sources with location $(x=40 / 9, y=4)$ and $(x=40 / 9, y=6)$, respectively. In both case a homogeneous initial value for both $u$ and $u_{t}$ are considered, i.e., $u(\mathbf{x}, t=0)=$ $u_{t}(\mathbf{x}, t=0)=0$. It is worthy to note that the reference solutions in 2-D examples are constructed using the same way as shown in 1-D example, which is obtained based on a global fine mesh, and in domain $\Omega_{2}$ only low frequency wave is included in the reference solution. 
The numerical results of the wave equation with external force $f_{1}$ are illustrated in Fig. 4, the results obtained from TGM and HIC are shown by the left and the right panel of Fig. 4, respectively. Comparing the results shown in the left and the right panel of Fig. 4 at the same time, we can clearly find that the spurious reflection due to the mesh size inhomogeneity interface between $\Omega_{1}$ and $\Omega_{2}$ is significantly reduced by using HIC method. More detailed information of this spurious reflection reduction can be founded in Fig. 5. The figures in left panel of Fig. 5 demonstrate the spatial distributions of difference between the TGM solution and the reference solution at various time, and the difference between the HIC solution and the reference solution at various time are shown in the right panel of Fig. 5. It can be more clearly observed that the reflection due to the interface in domain $\Omega_{1}$ is almost be completely diminished while there are still some wavy pattern in domain $\Omega_{2}$, which mainly comes from the approximation error as shown in Eq. (2.9). The mean square error of these two methods are plotted in Fig. 6. We could also find that HIC method can reduce the spurious reflection comparing to TGM.

The numerical results of the wave equation with external force $f_{2}$ are illustrated in Fig. 8. Similar to the first 2-D example. The results obtained from TGM and HIC are shown by the left and the right panel of Fig. 8, respectively. The spatial distributions of difference between the TGM solution and the HIC solution with respect to the reference solution at various time are shown in Fig. 9 and the total rMSE of both method are shown in Fig. 7. The observation from these figures show the phenomenon occurs in this example is almost the same as the previous example.

In general, comparing the corresponding results from Figs. 4 and 8, it can be seen that there is an obvious non-physical reflection at the interface when TGM is applied. The HIC method effectively reduce this phenomenon.

It is worthy to note that we can be found the spatial distributions of error in domain $\Omega_{2}$ have the contribution from both the low frequency component as well as the high frequency component in Figs. 5 and 9. We believe that this is because of the fact that the interface between the fine and the coarse mesh grid can not be simply considered as a perfect high frequency filter, i.e.,

$$
P f=\int_{\omega} 1_{|\omega|<A} \hat{f}(\omega) \exp (i \omega t) d \omega
$$

but should be treated as a more complicate operator $\tilde{P}$ which is hard to be implement in numerical calculation. However, in the implementation of our proposed HIC, we only need that projector to determinate $s_{0}$, which suggests the robustness of this method, that is, an exact formulation of $P$ is not necessary be needed in practice. The rMSE results in Figs. 6 and 7 also demonstrate the error component in $\Omega_{1}$ (Reflection) and $\Omega_{2}$ (Transmission) separately. We could clearly find that the error due to reflection is significantly reduced due to the introduce of HIC, which means the magnitude of spurious reflection is obviously eliminated. The transmission error due to the aliasing phenomenon is also reduced as shown in these figures. 


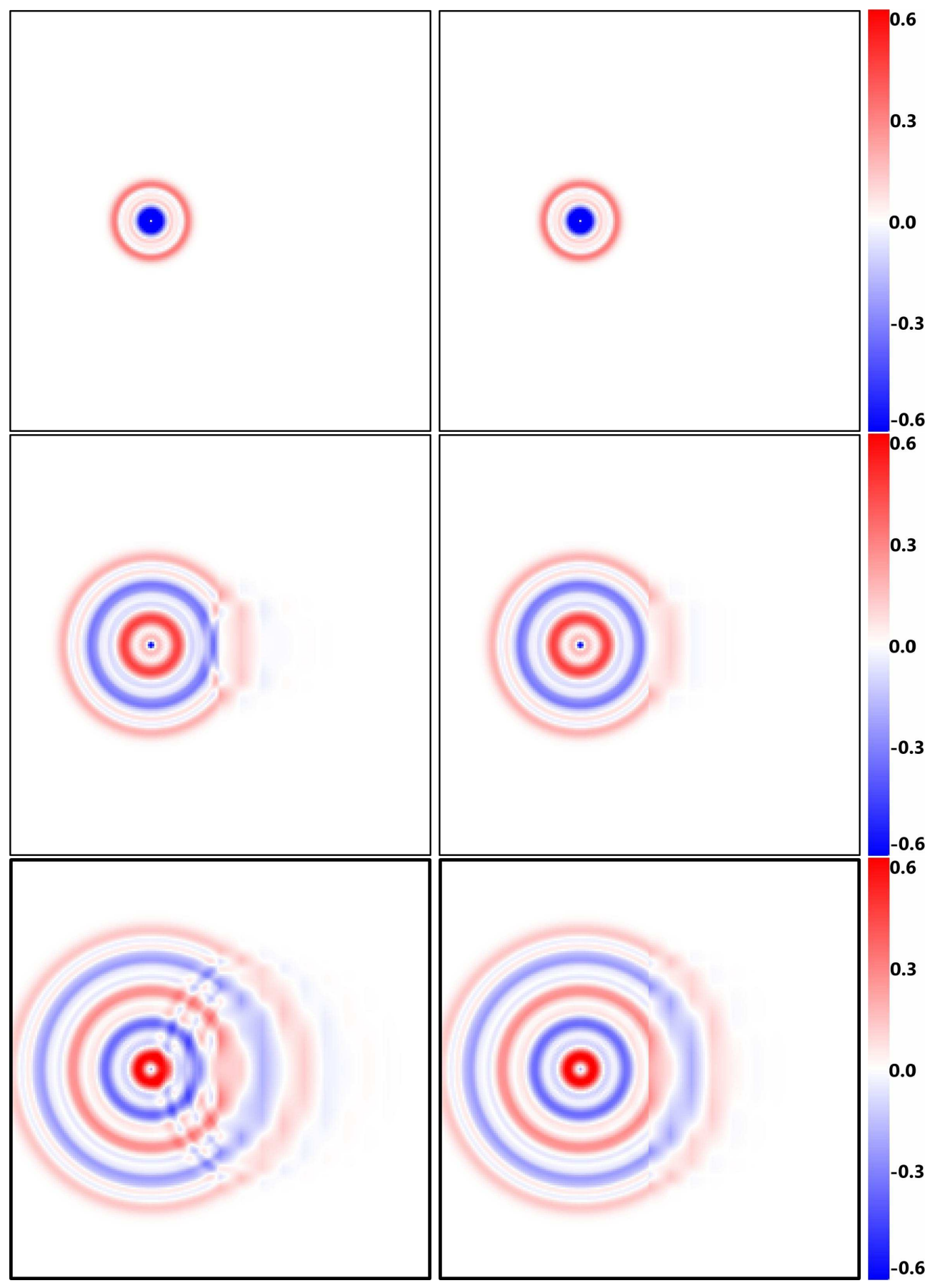

Figure 4: Results of 2-D example with external force $f_{1}$. From the top to the bottom is the evolution of time with $t=1, t=2.25, t=3.5$. The first column corresponds to the TGM while the second column to the HIC method. 


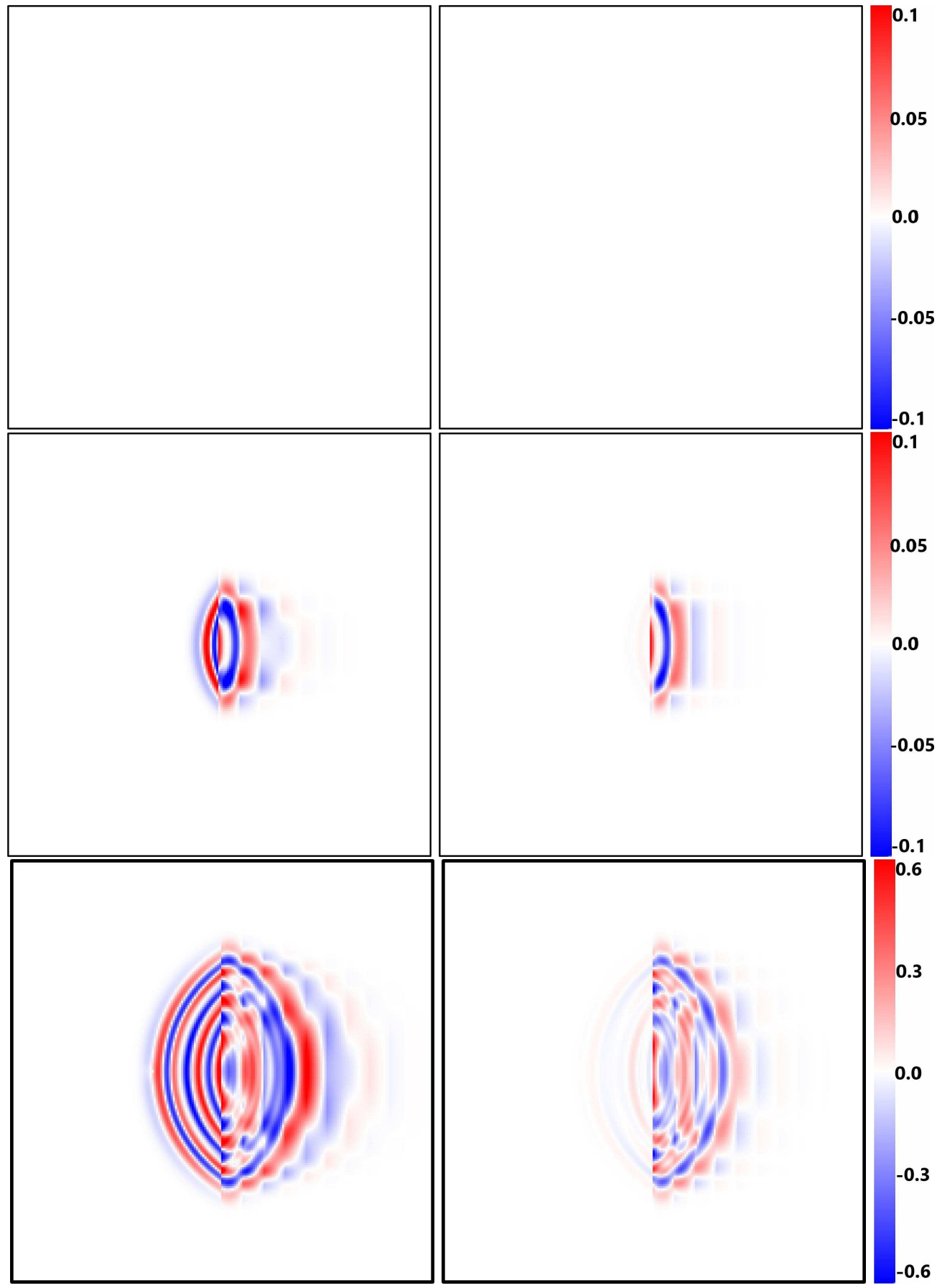

Figure 5: Spatial distribution between the numerical results obtained from TGM and HIC with respect to reference solution. From the top to the bottom is the evolution of time with $t=1, t=2.25, t=3.5$. The first column corresponds to the TGM while the second column to the HIC method. 


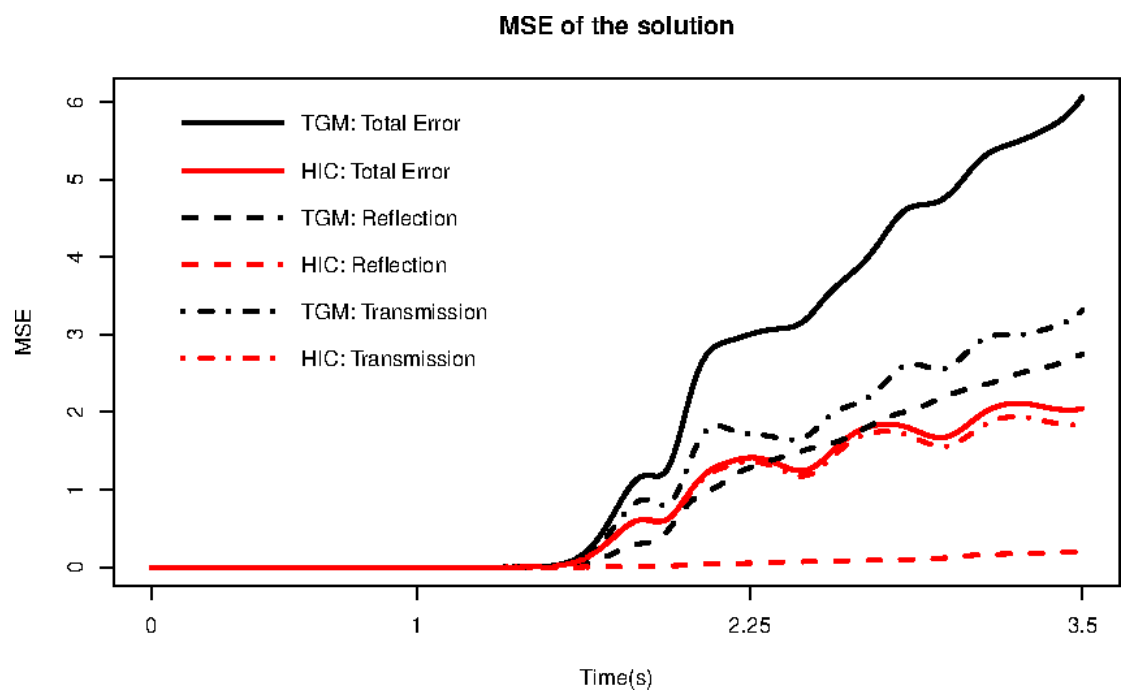

Figure 6: The relative mean square errors at different time for TGM and HIC.

Another mentionable point is that in Figs. 6 and 7 the mean square errors of the HIC method do not increase monotonously but with a periodical pattern. That comes from the application of PML. The choice of parameter $\lambda$ determines the absorbing ability of the boundary, which ends up with a result of HIC. However this problem can be covered on account of two reasons in practical: The $\lambda$ is independent with both the Lanczos process and the initial value, thus it can be searched out with a small calculation cost. And in our numerical experiment, $\lambda$ is not sensitive with the parameter used in HIC nor the incident wave.

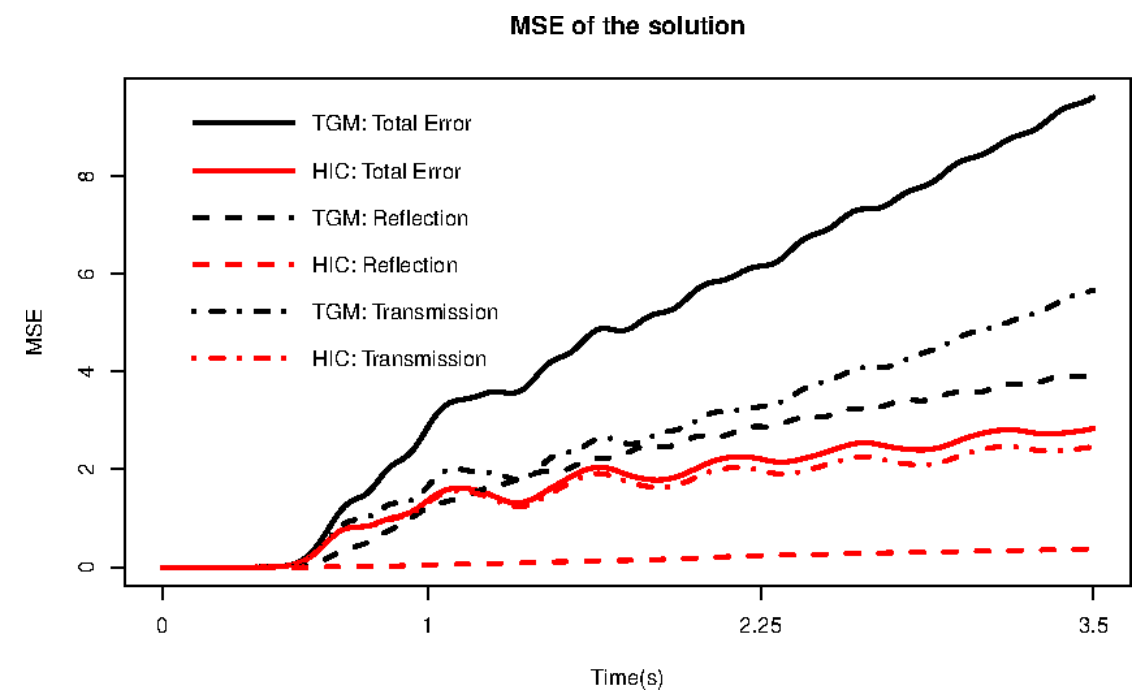

Figure 7: The relative mean square errors at different time for TGM and HIC. 


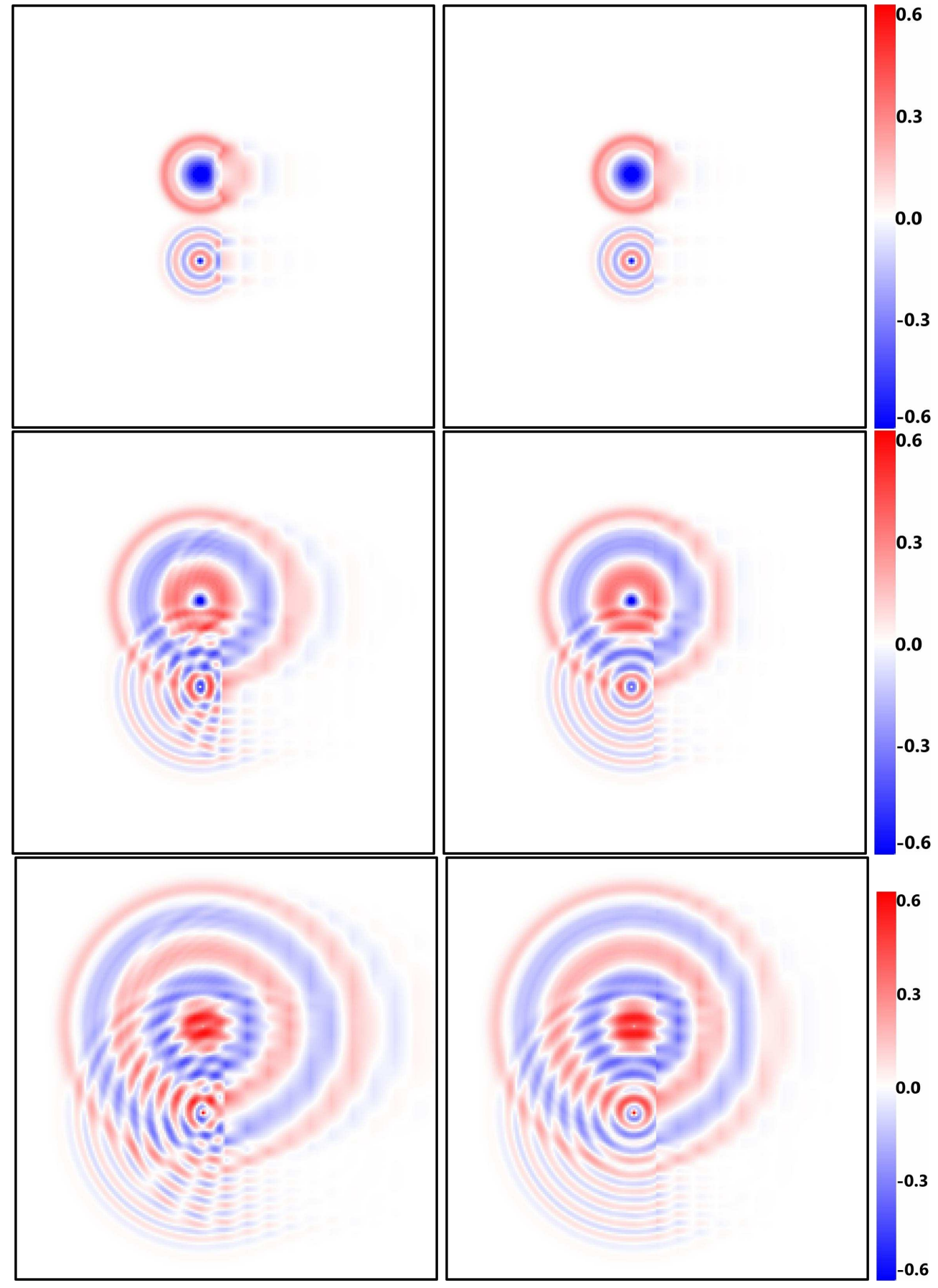

Figure 8: Results of 2-D example with external force $f_{2}$. From the top to the bottom is the evolution of time with $t=1, t=2.25, t=3.5$. The first column corresponds to the TGM while the second column to the HIC method. 


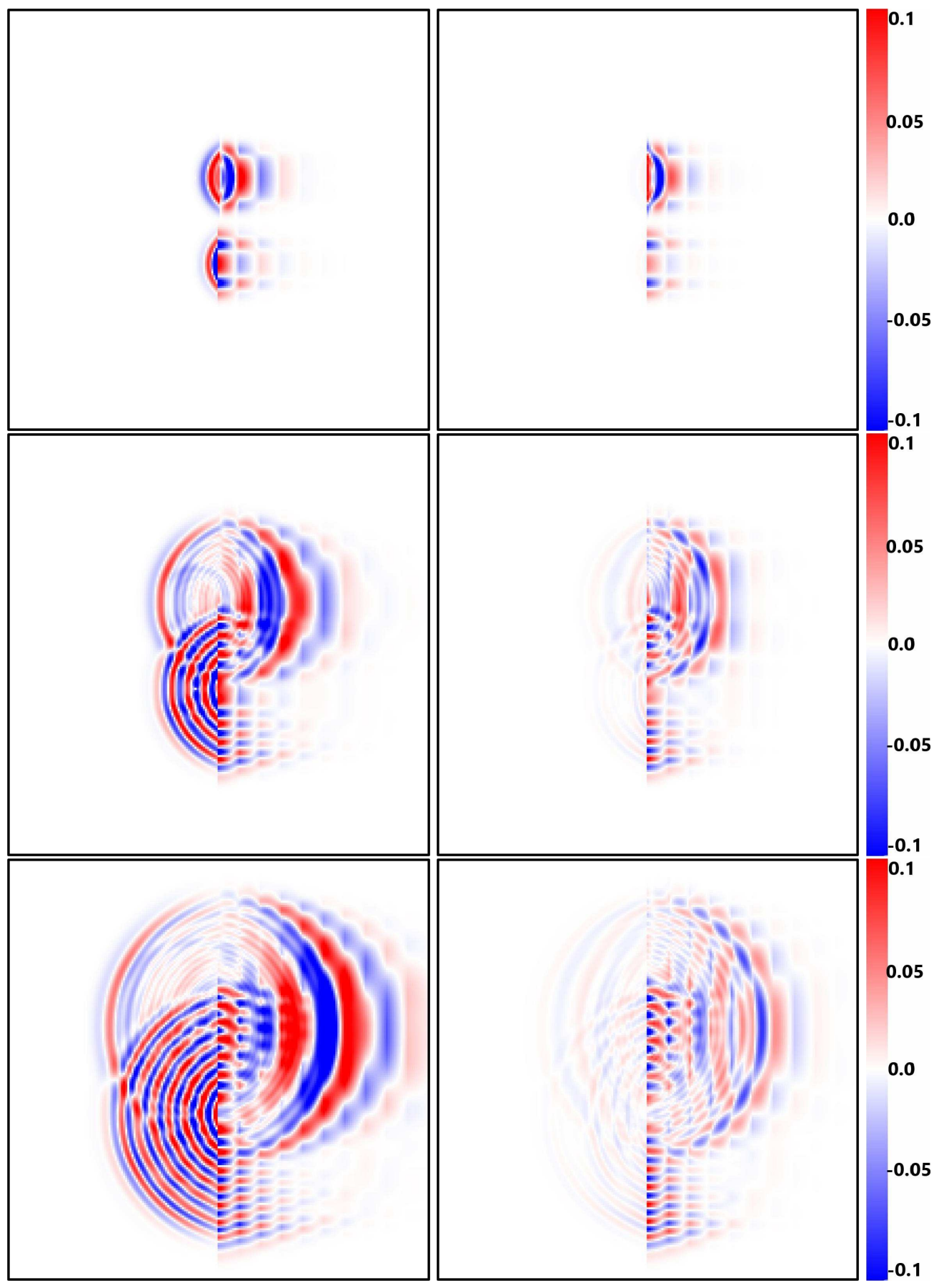

Figure 9: Spatial distribution between the numerical results obtained from TGM and HIC with respect to reference solution. From the top to the bottom is the evolution of time with $t=1, t=2.25, t=3.5$. The first column corresponds to the TGM while the second column to the HIC method. 


\section{Conclusion}

Our article focuses on the elimination of spurious reflection caused by the inhomogeneity of the space discretization. When a series of wave propagates through the interface between a domain with fine grid and a domain with coarse grid, the high frequency wave will be nonphysically reflected. Using the PVL method, we develop a new HIC method to eliminate this phenomenon. An auxiliary variable $\omega$ is introduced for the cancellation of the boundary source term which cause the spurious reflection, and one effective method based on Lanczos process are given to find this variable. The convergence of the HIC method are also acquired in our article. The numerical implementations are performed on both 1-D and 2-D domain with various external applied force. The results show in these examples clearly demonstrate the validity of our proposed HIC method in spurious reflection elimination.

\section{Acknowledgments}

This work is supported by the National Key Research and Development Program of China (Grant No. 2020YFA0714200), by the National Natural Science Foundation of China (Grants No. 12125103, 12071362, 12101062), by China Postdoctoral Science Foundation (Grant No. 2019M660558), and by the Natural Science Foundation of Hubei Province (Grant No. 2019CFA007). Numerical calculations are carried out on the supercomputing system of the Supercomputing Center of the Wuhan University.

\section{References}

[1] Z. BAI, Krylov subspace techniques for reduced-order modeling of large-scale dynamical systems, Appl. Numer. Math. 43 (2002), 9-44.

[2] Z. P. BAŽANT, Spurious reflection of elastic waves in nonuniform finite element grids, Comput. Methods Appl. Mech. Engrg. 16 (1978), 91-100.

[3] Z. P. BAŽANT AND Z. CELEP, Spurious reflection of elastic waves in nonuniform meshes of constant and linear strain unite elements, Computers Structures, 15 (2016), 451-459.

[4] M. J. BERgER AND J. Oliger, Adaptive mesh refinement for hyperbolic partial differential equations, J. Comput. Phys. 53 (1984), 484-512.

[5] D. L. Boley, Krylov space methods on state-space control models, Circuits, Syst. Signal Process. 13 (1994), 733-758.

[6] L. Brillouin, Wave Propagation in Periodic Structures, Dover Publications, Ins., 1953.

[7] Z. CELEP AND Z. P. BAŽANT, Spurious reflection of elastic waves due to gradually changing finite element size, Internat. J. Numer. Methods Engrg. 19 (1983), 631-646.

[8] W. E AND Z. HUANG, Matching conditions in atomistic-continuum modeling of materials, Phys. Rev. Lett. 87 (2001), 135501.

[9] W. E AND Z. HUANG, A dynamic atomistic-continuum method for the simulation of crystalline materials, J. Comput. Phys. 182 (2002), 234-261.

[10] P. FELDMANN AND R. W. FREUND, Efficient linear circuit analysis by pade approximation via the Lanczos process, IEEE Trans. Comput.-Aided Des. Integr. Circuits Syst 14 (1995), 639-649. 
[11] K. Gallivan, E. GRimme, And P. V. Dooren, Asymptotic waveform evaluation via a Lanczos method, Appl. Math. Lett. 7 (1994), 75-80.

[12] W. B. GRAGG, Matrix interpretation and applications of the continued fraction algorithm, Rocky Mountain J. Math. 4 (1974), 213-226.

[13] A. IDESMAN AND B. DEY, Ptimal reduction of numerical dispersion for wave propagation problems. Part 2: Application to 2-D isogeometric elements, Comput. Meth. Appl. Mech. Engng. 321 (2017), 235-268.

[14] S. KULKARNI AND A. TABARRAEI, An analytical study of wave propagation in a peridynamic bar with nonuniform discretization, Eng. Fract. Mech. 190 (2017), 347-366.

[15] S. KulKarni, A. TABARRAeI, AND X. WANG, Study of spurious wave reflection at the interface of peridynamics and finite element regions, Proceedings of the ASME 2018 International Mechanical Engineering Congress and Exposition. Volume 9: Mechanics of Solids, Structures, and Fluids, (2018), V009T12A054.

[16] J. LEI AND R. J. ROGERS, Spurious wave reflections at an interface of different physical properties in finite-element wave solutions, Commun. numer. methods eng. 7 (2010), 595602.

[17] S. Li, X. LiU, A. Agrawal, AND A. C. To, Perfectly matched multiscale simulations for discrete lattice systems: Extension to multiple dimensions, Phys. Rev. B, 74 (2006), 045418.

[18] X. LI, A coarse-grained molecular dynamics model for crystalline solids, Internat. J. Numer. Methods Engrg. 83 (2010), 986-997.

[19] X. LI, Coarse-graining molecular dynamics models using an extended Galerkin projection method, Internat. J. Numer. Methods Engrg. (2014).

[20] X. LI, J. Z. YANG, AND W. E, A multiscale coupling method for the modeling of dynamics of solids with application to brittle cracks, J. Comput. Phys. 229 (2010), 3970-3987.

[21] C. G. MAKRIDAKIS, Finite element approximations of nonlinear elastic waves, Math. Comp. 61 (1993), 569-594.

[22] S. OSHER AND R. SANDERS, Numerical approximations to nonlinear conservation laws with locally varying time and space grids, Math. Comp. 41 (1983), 321-336.

[23] L. T. Pillage and R. A. Rohrer, Asymptotic waveform evaluation for timing analysis, IEEE Trans. Comput.-Aided Des. Integr. Circuits Syst 9 (2002), 352-366.

[24] R. D. Slone AND R. LeE, Applying Padé via Lanczos to the finite element method for electromagnetic radiation problems, Radio Science 35 (2000), 331-340.

[25] Z. TAN, Z. ZHANG, Y. HuANG, AND T. TANG, Moving mesh methods with locally varying time steps, J. Comput. Phys. 200 (2004), 347-367.

[26] S. TANG, T. Y. HOU, AND W. K. LIU, A mathematical framework of the bridging scale method, Internat. J. Numer. Methods Engrg. 65 (2006), 1688-1713.

[27] A. C. To And S. LI, Perfectly matched multiscale simulations, Phys. Rev. B 72 (2005), 035414.

[28] G. J. WAGNER AND W. K. LiU, Coupling of atomistic and continuum simulations using a bridging scale decomposition, J. Comput. Phys. 190 (2003), 249-274. 